\title{
Antimicrobial Efficacy of 3 Oral Antiseptics Containing Octenidine, Polyhexamethylene Biguanide, or Citroxx: Can Chlorhexidine Be Replaced?
}

\author{
Nadine Rohrer, DDS; Andreas F. Widmer, MD, MS; Tuomas Waltimo, DDS; Eva M. Kulik, PhD; Roland Weiger, DDS; \\ Elisabeth Filipuzzi-Jenny, MT; Clemens Walter, DDS
}

\begin{abstract}
BACKGROUND. Use of oral antiseptics decreases the bacterial load in the oral cavity.
овлестіле. To compare the antimicrobial activity of 3 novel oral antiseptics with that of chlorhexidine, which is considered the "gold standard" of oral hygiene.
\end{abstract}

DESIGN. Comparative in vitro study.

METHODs. Four common oral microorganisms (Streptococcus sanguinis, Streptococcus mutans, Candida albicans, and Fusobacterium nucleatum) were tested under standard conditions and at different concentrations, by use of a broth dilution assay and an agar diffusion assay and by calculating the $\log _{10}$ reduction factor (RF). The antimicrobial activity of each antiseptic was assessed by counting the difference in bacterial densities (ie, the $\log _{10}$ number of colony-forming units of bacteria) before and after the disinfection process.

RESULTS. The oral antiseptics containing octenidine (with an RF in the range of 7.1-8.24 CFU/mL) and polyhexamethylene biguanide (with an RF in the range of 7.1-8.24 CFU/mL) demonstrated antimicrobial activity comparable to that of chlorhexidine (with an RF in the range of 1.03-8.24 CFU/mL), whereas the mouth rinse containing Citroxx (Citroxx Biosciences; with an RF in the range of 0.22-1.36 $\mathrm{CFU} / \mathrm{mL}$ ) showed significantly weaker antimicrobial efficacy. Overall, octenidine and polyhexamethylene biguanide were more active at lower concentrations.

CONCLUSION. Oral antiseptics containing the antimicrobial agent octenidine or polyhexamethylene biguanide may be considered as potent alternatives to chlorhexidine-based preparations.

Infect Control Hosp Epidemiol 2010; 31(7):733-739

The mouth and oropharynx are colonized with microorganisms, which include gram-negative anaerobic bacteria, Staphylococcus aureus, and Candida species. The most common oral infections associated with bacteria are diseases of the tooth-supporting structures (ie, gingivitis and periodontitis), affecting up to $98 \%$ of adults in the United States. ${ }^{1}$ In addition, these microorganisms have the ability to invade the bloodstream, resulting in transient bacteremia, especially during tooth brushing and flossing ( $20 \%-68 \%$ of cases) and even during the chewing of food ( $7 \%-51 \%$ of cases). The microorganisms found in the oral cavity are also associated with pneumonia. ${ }^{2,3} \mathrm{~A}$ meta-analysis by Chan et $\mathrm{al}^{4}$ provided indirect evidence of the impact of oral microorganisms; they showed that oral decontamination with an antiseptic administered as prophylaxis reduces the incidence of ventilatorassociated pneumonia. Similarly, a randomized controlled clinical trial ${ }^{5}$ showed that selective oral decontamination re- sulted in a significant reduction in cases of ventilator-associated pneumonia.

Chlorhexidine is a cationic biguanide that was introduced as an antimicrobial agent by G. E. Davies in $1954 .{ }^{6}$ Because of the antimicrobial spectrum and the suspected remnant effect, chlorhexidine digluconate is considered the "gold standard" of oral hygiene in the United States. However, the use of chlorhexidine-containing mouth rinses can result in adverse effects, such as taste disturbance or tooth staining, that may have an impact on compliance. In addition to the cytotoxicity of chlorhexidine on corneal and endothelial cells, the neurotoxicity of chlorhexidine was observed in animal models, which prevents it from being used for very long. ${ }^{8,9}$ Allergic reactions are common if chlorhexidine is applied in concentrations of more than $4 \%$, and serious anaphylactic reactions have been described. ${ }^{10} \mathrm{~A}$ higher concentration of chlorhexidine would be preferable for mouth rinses because

From the School of Dentistry, University of Basel (N.R., T.W., E.M.K., R.W., E.F.-J., C.W.), and the Department of Infectious Diseases and Hospital Epidemiology, University Hospital Basel (A.F.W.), Basel, Switzerland.

Received November 16, 2009; accepted January 29, 2010; electronically published June 1, 2010.

(C) 2010 by The Society for Healthcare Epidemiology of America. All rights reserved. 0899-823X/2010/3107-0011\$15.00. DOI: 10.1086/653822 
they are diluted after application. However, there is evidence that the antimicrobial effect of chlorhexidine is primarily bacteriostatic, rather than bactericidal, and that the antimicrobial effect of chlorhexidine is probably overestimated because of lack of neutralization after sampling. ${ }^{11}$ In addition, its efficacy against several gram-negative pathogens is limited.

In Europe, additional oral antimicrobial mouth rinses containing octenidine, polyhexamethylene biguanide, or Citroxx (Citroxx Biosciences) have been marketed that claim to have a similar antimicrobial spectrum but are associated with fewer adverse effects, less toxicity, and less frequency of allergic reactions. ${ }^{12,13}$ Citroxx consists of a patented blend of bioflavinoids and fruit acids. ${ }^{14}$ Citroxx-containing solutions are frequently used as active ingredients in a variety of applications, cosmetics, personal care products, or hand washes.

In addition to polyhexamethylene biguanide's antibacterial activity against oral microorganisms, ${ }^{13}$ it has been used in antimicrobial gauze dressing to prevent surgical site infections due to methicillin-resistant $S$. aureus..$^{15}$ Octenidine may be used for prolonged periods of time because it is associated with fewer adverse effects, compared with chlorhexidine. In the clinical setting, octenidine has been successfully used for the prevention of catheter-related infections. ${ }^{12,16}$

A standardized follow-up period, which included laboratory analysis of sessile and planktonic bacteria, was suggested for the evaluation of the antimicrobial activities of oral antiseptics. ${ }^{17,18}$ Finally, the therapeutic efficacy of each antiseptic has to be evaluated in clinical studies (ie, with the use of phase 3 tests).

However, to our knowledge, there have been no side-byside in vitro studies evaluating the antimicrobial efficacy of these 3 oral antiseptics, unlike those evaluating the antimicrobial efficacy of chlorhexidine. In addition, concerns have been raised about the emergence of microorganisms resistant to chlorhexidine, which appears not to be a clinical problem yet. The aim of the present in vitro study was to compare the antimicrobial activity of 3 commercially available, novel oral antiseptics (containing octenidine, polyhexamethylene biguanide, or Citroxx, respectively) with that of chlorhexidine.

\section{METHODS}

\section{Microorganisms and Growth Conditions}

The DSM 20068 strain of Streptococcus sanguinis and the DSM 20523 strain of Streptococcus mutans were obtained from the Deutsche Sammlung von Mikroorganismen und Zellkulturen, and the ATCC 90028 strain of Candida albicans and the ATCC 10953 strain of Fusobacterium nucleatum were obtained from the American Type Culture Collection. The strains were grown on Columbia blood agar plates (BBL; Becton Dickinson) supplemented with $50 \mathrm{~mL} / \mathrm{L}$ of human blood, $0.5 \mathrm{mg} /$ $\mathrm{L}$ of menadione, and $5 \mathrm{mg} / \mathrm{L}$ of hemin at $37^{\circ} \mathrm{C}$. C. albicans was incubated under aerobic conditions for 24 hours; S. mutans was incubated in air with $10 \% \mathrm{CO}_{2}$ for 48 hours; $S$. sanguinis was incubated under anaerobic conditions with $10 \%$ $\mathrm{CO}_{2}, 10 \% \mathrm{H}_{2}$, and $80 \% \mathrm{~N}_{2}$ for 24 hours; and $F$. nucleatum was incubated under anaerobic conditions for 48 hours. These conditions were used throughout our study.

\section{Preparation of Test Solutions}

The following 3 commercially available oral antiseptics were compared: Octenidol (Schülke \& Mayr AG), which contains octenidine as an antimicrobial agent; ProntOral (B. Braun Medical), which contains polyhexamethylene biguanide as an antimicrobial agent; and OralClens (Oraldent), which contains Citroxx as an antimicrobial agent. The exact formulas for these mouth rinses are not in the public domain because of patent-related restrictions. Chlorhexidine digluconate solution in a frequently used and recommended concentration (0.2\%) (Sigma) served as a comparator. These commercially available mouth rinses were applied in their recommended concentration and also at serial dilutions down to $10^{-3}$ to test lower concentrations as they may occur during application.

\section{Broth Dilution Assay}

The test strains were suspended in $0.9 \% \mathrm{NaCl}$, harvested by centrifugation at 10,000 rpm (radius, $85 \mathrm{~mm}$ ) for 5 minutes, and resuspended in $0.9 \% \mathrm{NaCl}$. The microbial suspensions were adjusted to a final cell density of approximately $6 \times$ $10^{8} \mathrm{CFU} / \mathrm{mL}$, controlled by plating appropriate dilutions of the suspensions onto the Columbia blood agar plates. An aliquot of $100 \mu \mathrm{L}$ of a microbial suspension was added to $900 \mu \mathrm{L}$ of a test solution and mixed thoroughly. After incubation at room temperature for 1 minute, the suspensions were serially diluted in $0.9 \% \mathrm{NaCl}$, and appropriate dilutions were plated onto Columbia blood agar media. The number of colony-forming units per milliliter was determined after strain-specific incubation, and the mean $\log _{10}$ CFU reduction factor was calculated. The antimicrobial activity of each antiseptic was assessed by counting the difference in bacterial densities (ie, the $\log _{10}$ number of colony-forming units of bacteria) before and after the disinfection process. All experiments were performed 3 times.

\section{Agar Diffusion Assay}

A modified agar diffusion assay was used. ${ }^{19}$ In brief, $20 \mu \mathrm{L}$ of undiluted or diluted mouth rinse was placed onto a blank, nonimpregnated filter-paper disk (bioMérieux; diameter, 6 $\mathrm{mm}$ ). Sterilized $0.9 \% \mathrm{NaCl}$ served as a negative control. Suspensions equal to the $0.5 \mathrm{McF}$ arland standard for each bacterial strain and suspensions equal to 2-4 McFarland for $C$. albicans were prepared in broth medium: Sabouraud dextrose broth (Becton Dickinson) for C. albicans, Schaedler broth (Becton Dickinson) for S. mutans and S. sanguinis, and thioglycolate broth (Becton Dickinson) for $F$. nucleatum. After inoculating the suspensions evenly with a cotton swab onto Columbia blood agar plates, the disks were placed and gently 
pressed on the plates with sterile forceps. After strain-specific incubation, all plates were photographed, and the inhibition zones were measured. All experiments were performed 3 times.

\section{Statistical Analyses}

All analyses were done by use of the statistical package $\mathrm{R}$, version 2.8 (The R Foundation for Statistical Computing). Prior to the statistical analyses, all colony-forming units per milliliter were log-transformed. Statistical comparisons were made for the results obtained for the undiluted mouth rinses. For each strain, linear regression models were performed, with "diameter" and "count reduction" as dependent factors and "treatment" as the independent factor. Pairwise differences in the mean values were calculated between each treatment for each strain independently. Ninety-five percent confidence intervals and 2 -sided $P$ values were calculated. $P$ values of less than 0.05 were considered to be statistically significant. $P$ values were adjusted for multiple comparisons according to the Tukey method. Box plots were prepared that show the dependency of the count reduction and the inhibition zone on the treatment and concentration of the test solutions. The efficacy of a mouth rinse was calculated as a $\log _{10}$ reduction factor after the mouth rinse was exposed for 1 minute in a broth medium.

\section{RESULTS}

In general, the antimicrobial activity of the mouth rinses containing octenidine and polyhexamethylene biguanide was comparable to that of chlorhexidine. In contrast, the mouth rinse containing Citroxx showed remarkably weaker antimicrobial efficacy. Dose-dependent killing was observed in all experiments, whereas the cell counts of the negative control (ie, $0.9 \% \mathrm{NaCl}$ ) remained unaffected.

\section{S. sanguinis}

In both the broth dilution assay (Figure $1 A$ ) and the agar diffusion assay (Figure $1 B$ ), similar antimicrobial activity against $S$. sanguinis was demonstrated by Octenidol and ProntOral. The broth dilution assay revealed that Octenidol and ProntOral were active against $S$. sanguinis even at very low concentrations, whereas chlorhexidine demonstrated only a moderate effect. In contrast, the undiluted chlorhexidine was the most effective solution in the agar diffusion assay. In the agar disk assay, the disks treated with undiluted OralClens showed an inhibition zone comparable to the disks treated with undiluted Octenidol or undiluted ProntOral. In the broth dilution assay, no antimicrobial efficacy was evident for OralClens (mean reduction factor, $0.26 \mathrm{CFU} / \mathrm{mL}$ ). The mean reduction factor was $7.2 \mathrm{CFU} / \mathrm{mL}$ for undiluted $\mathrm{Oc}$ tenidol, 7.2 CFU/mL for undiluted ProntOral, and 1.03 CFU/ $\mathrm{mL}$ for undiluted chlorhexidine. In the broth dilution assay, there were statistically significantly lower microbial counts for chlorhexidine $(P<.001)$ and OralClens $(P<.001)$, compared with ProntOral and Octenidol. The diameters of the inhibition zones were statistically significant for Octenidol, compared with chlorhexidine $(P<.001)$ or ProntOral $(P<$
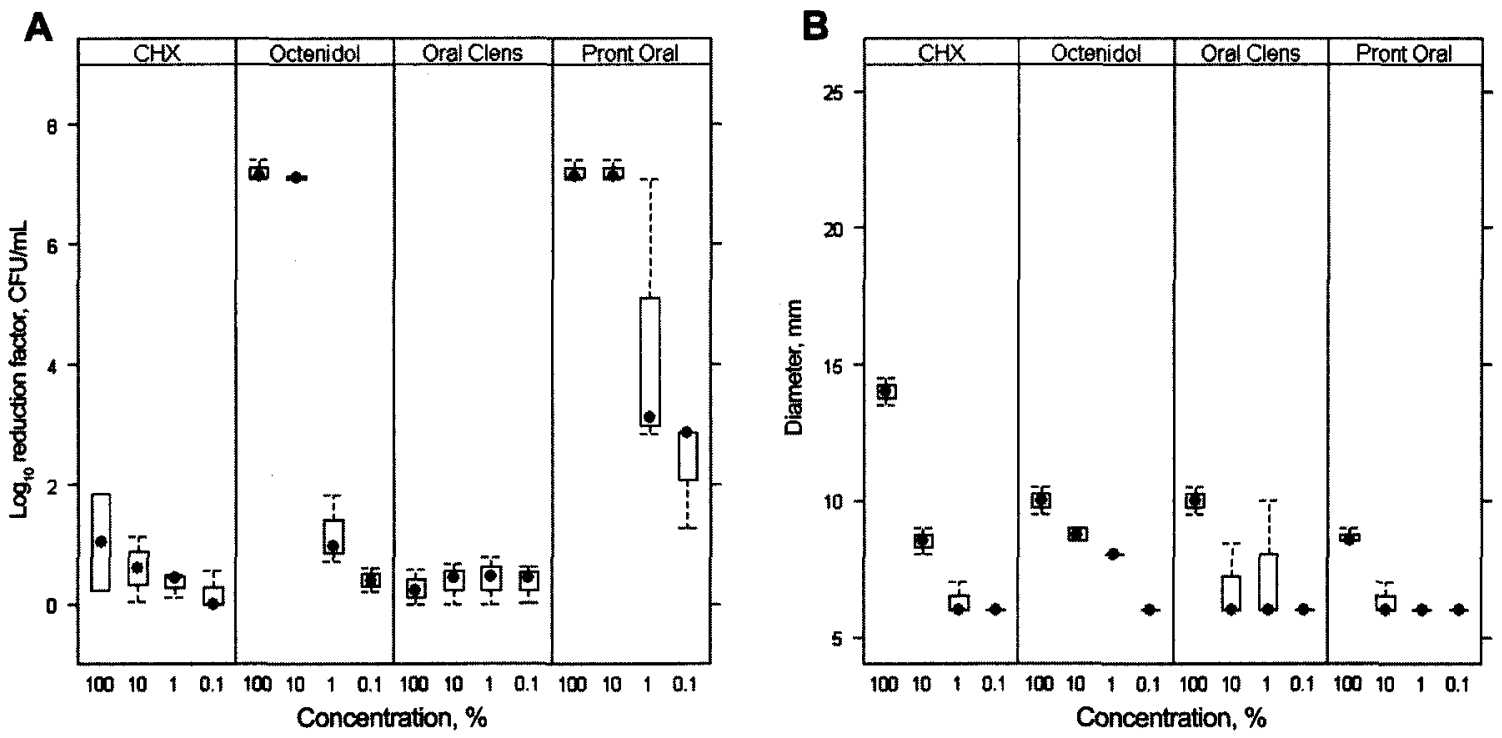

FIGURE 1. Box plot summarizing the $\log _{10}$ reduction of total microbial counts (ie, the reduction factor, in units of CFU/mL) and the diameters of the inhibition zones obtained when testing different concentrations of the mouth rinses against Streptococcus sanguinis. The point represents the mean value. The top and bottom borders of the box represent the 75 th and 25 th percentiles, respectively. The whiskers above and/or below the box represent the 90th and 10th percentiles. $\mathrm{CHX}$, chlorhexidine. 

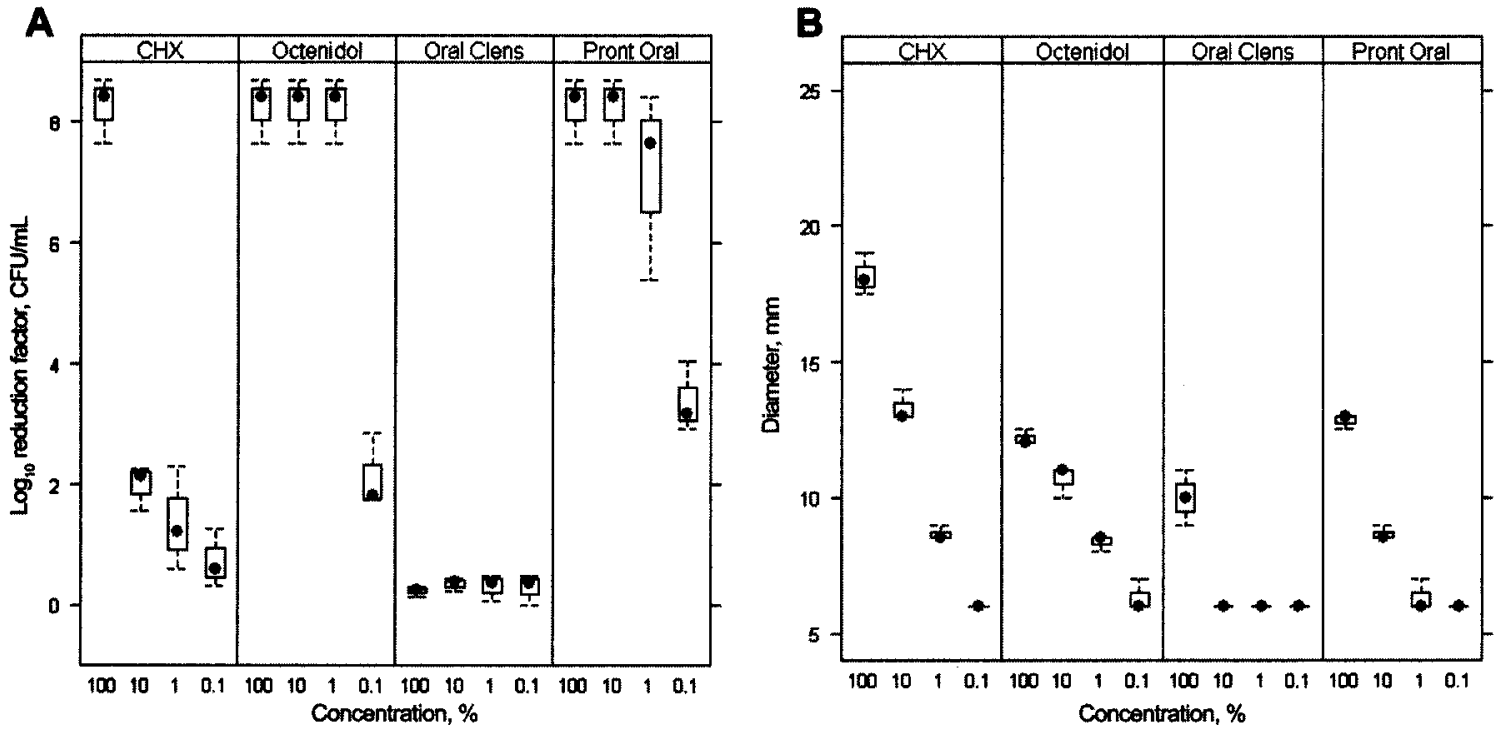

FIGURE 2. Box plot summarizing the $\log _{10}$ reduction of total microbial counts (ie, the reduction factor, in units of CFU/mL) and the diameters of the inhibition zones obtained when testing different concentrations of the mouth rinses against Streptococcus mutans. The point represents the mean value. The top and bottom borders of the box represent the 75th and 25th percentiles, respectively. The whiskers above and/or below the box represent the 90th and 10th percentiles. CHX, chlorhexidine.

$.03)$; for OralClens, compared with ProntOral $(P<.03)$; and for chlorhexidine, compared with OralClens $(P<.001)$.

\section{S. mutans}

S. mutans was more susceptible to chlorhexidine, Octenidol, and ProntOral than was S. sanguinis. Concentrations of Oc- tenidol and ProntOral as low as $1 \%$ were able to kill this microorganism in the broth dilution assay (Figure $2 A$ ). As with $S$. sanguinis, no reduction in the number of colonyforming units per milliliter could be detected when $S$. mutans was treated with OralClens, whereas modest inhibitory activity was shown in the agar diffusion assay (Figure $2 B$ ). The
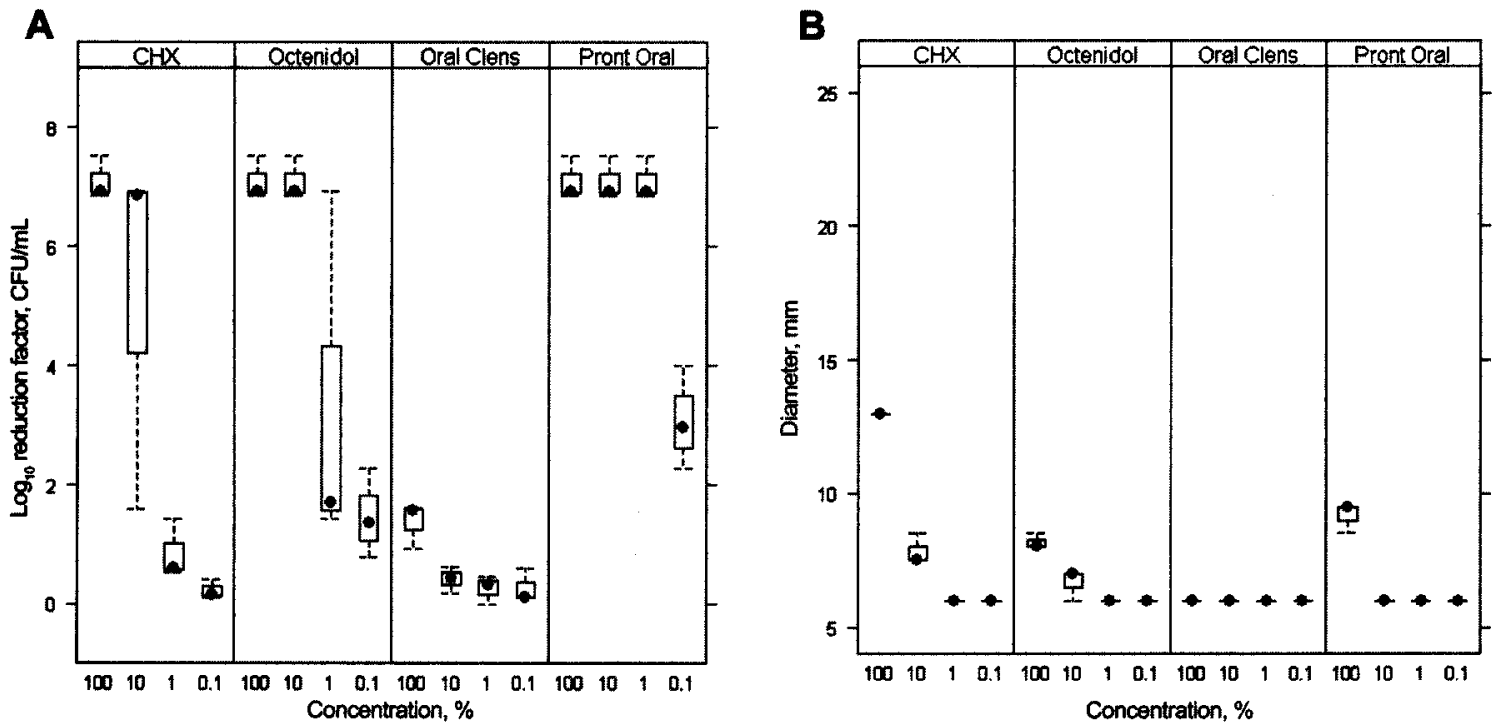

FIGURE 3. Box plot summarizing the $\log _{10}$ reduction of total microbial counts (ie, the reduction factor, in units of $\mathrm{CFU} / \mathrm{mL}$ ) and the diameters of the inhibition zones obtained when testing different concentrations of the mouth rinses against Fusobacterium nucleatum. The point represents the mean value. The top and bottom borders of the box represent the 75 th and 25 th percentiles, respectively. The whiskers above and/or below the box represent the 90th and 10th percentiles. $\mathrm{CHX}$, chlorhexidine. 

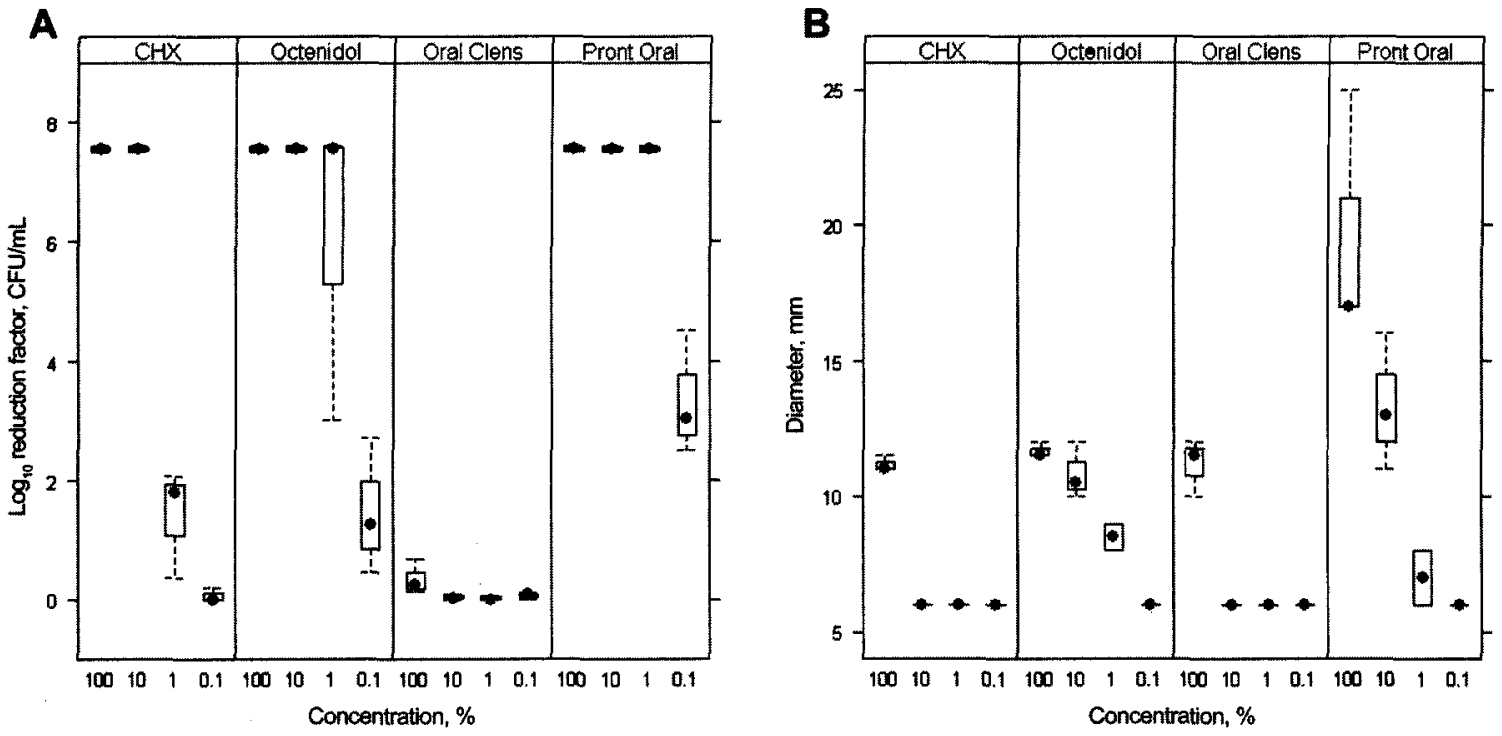

FIGURE 4. Box plot summarizing the $\log _{10}$ reduction of total microbial counts (ie, the reduction factor, in units of CFU/mL) and the diameters of the inhibition zones obtained when testing different concentrations of the mouth rinses against Candida albicans. The point represents the mean value. The top and bottom borders of the box represent the 75 th and 25 th percentiles, respectively. The whiskers above and/or below the box represent the 90th and 10th percentiles. CHX, chlorhexidine.

mean reduction factor was $8.24 \mathrm{CFU} / \mathrm{mL}$ for undiluted Octenidol, 8.24 CFU/mL for undiluted ProntOral, 0.22 CFU/ $\mathrm{mL}$ for undiluted OralClens, and $8.24 \mathrm{CFU} / \mathrm{mL}$ for undiluted chlorhexidine. A statistically significant difference in the reduction in microbial counts with chlorhexidine, Octeni$\mathrm{dol}$, and ProntOral, compared with OralClens $(P<.001)$, was found using the highest concentration of the mouth rinses in the broth dilution assay. In the agar diffusion assay, the inhibition zone for chlorhexidine was statistically significantly different from the inhibition zones for OralClens $(P<.001)$, Octenidol $(P<.001)$, and ProntOral $(P<.001)$. The inhibition zones for Octenidol $(P=.02)$ and ProntOral $(P<$ $.001)$ were statistically significantly different from the inhibition zone for OralClens.

\section{F. nucleatum}

Both the broth dilution assay (Figure $3 A$ ) and the agar diffusion assay (Figure $3 B$ ) showed that Octenidol, ProntOral, and chlorhexidine are effective against $F$. nucleatum. ProntOral demonstrated remarkable antimicrobial efficacy even at the lowest concentration in the broth dilution assay. Chlorhexidine and Octenidol were also potent against $F$. nucleatum, whereas OralClens showed only a minor killing effect in the broth dilution assay and failed to inhibit growth in the agar diffusion assay. The mean reduction factor was 7.1 $\mathrm{CFU} / \mathrm{mL}$ for undiluted Octenidol, $7.1 \mathrm{CFU} / \mathrm{mL}$ for undiluted ProntOral, $1.36 \mathrm{CFU} / \mathrm{mL}$ for undiluted OralClens, and 7.1 CFU/mL for undiluted chlorhexidine. Compared with OralClens, when the highest concentrations of chlorhex- idine $(P<.001)$, Octenidol $(P<.001)$, and ProntOral $(P<$ .001 ) were used in the broth dilution assay, the reductions in microbial counts were statistically significant. The diameters of the inhibition zones on the disks treated with chlorhexidine, OralClens, Octenidol, and ProntOral differed statistically significantly in the agar diffusion assay, depending on whether the highest concentration of the mouth rinses or the chlorhexidine control solution was used (OralClens vs chlorhexidine, ProntOral, and Octenidol $[P<.001]$; Octenidol vs ProntOral $[P=.02]$; chlorhexidine vs Octenidol and ProntOral $[P<.001])$.

\section{C. albicans}

ProntOral, Octenidol, and chlorhexidine were effective even at low concentrations in the broth dilution assay (Figure $4 A$ ) and in the agar diffusion assay (Figure $4 B$ ). Only the undiluted OralClens showed inhibition in the agar diffusion assay. The mean reduction factor was $7.54 \mathrm{CFU} / \mathrm{mL}$ for undiluted Octenidol, 7.54 CFU/mL for undiluted ProntOral, 0.35 CFU/ $\mathrm{mL}$ for undiluted OralClens, and $7.54 \mathrm{CFU} / \mathrm{mL}$ for undiluted chlorhexidine. Statistically significant differences in the reduction in microbial counts with chlorhexidine, OralClens, and ProntOral, compared with OralClens $(P<.001)$, were found using the highest concentrations of the solutions in the broth dilution assay. In the agar diffusion assay, statistically significant differences were found for disks treated with ProntOral, compared with OralClens $(P=.01)$, Octenidol $(P=.01)$, and chlorhexidine $(P=.01)$. 


\section{DISCUSSION}

Our in vitro study clearly demonstrated that the efficacy of the mouth rinses containing octenidine or polyhexamethylene biguanide (but not Citroxx) was to similar the efficacy of chlorhexidine. Octenidine and polyhexamethylene biguanide remained very active even when highly diluted. In contrast, chlorhexidine lost its antimicrobial efficacy when diluted to less than $10 \%$ of its original concentration. Therefore, it is conceivable that such mouth rinses are at least as effective as chlorhexidine, on basis of these in vitro experiments. In fact, these products may be even more effective than chlorhexidine, because many-fold dilutions are to be expected in mouth rinses that are used by the general public. Similar to chlorhexidine, octenidine and polyhexamethylene biguanide have prolonged effects after application.

The oral cavity is the natural habitat of a wide variety of microorganisms. ${ }^{20}$ Some of them may be beneficial to the host, while others are considered to play a role in the pathogenesis of certain oral or other infectious diseases, including ventilator-associated pneumonia. ${ }^{20-23}$ Ideally, microorganisms beneficial to the host should not be affected by an antiseptic. The selection of the species in the present study represents some of the microorganisms with documented key characteristics in the oral habitat or for the pathogenesis of pleuropulmonary infections. S. sanguinis is commonly found in the oral cavity, is not considered pathogenic, and may be beneficial to the host. This bacterium is thought to demonstrate a competitive inhibition of $S$. mutans, ${ }^{24,25}$ whereas $S$. mutans is closely related to the pathogenesis of caries. ${ }^{22} F$. nucleatum, a gram-negative anaerobic rod, is an important link between the primary and secondary colonizers in the dental plaque and is detected in chronic periodontitis lesions and frequently recovered from patients with necrotizing-ulcerative gingivitis and/or periodontitis. ${ }^{23,26}$ In contrast to the treatment of chronic periodontitis, emergency treatment of necrotizing-ulcerative gingivitis and/or periodontitis consists of mechanical debridement with adjunctive use of antiseptics. F. nucleatum may also cause ventilator-associated pneumonia or other severe infections. ${ }^{2,27}$ The yeast $C$. albicans lives in a state of commensalism in the oral cavity but is also an important opportunistic pathogen that can cause infections varying from harmless newborn thrush to severe deep mucocutaneous candidiasis or even sepsis in patients with a local or systemic compromised immune response or in patients who take antibiotics. ${ }^{28-30}$

Our investigation has revealed that the killing efficacy of chlorhexidine against $S$. sanguinis was weaker than that of mouth rinses containing octenidine or polyhexamethylene biguanide (Figure 1). This finding is in accordance with that of Decker et al. ${ }^{31}$ In ascending order, the following mouth rinses were found to be the most effective against $S$. mutans: those containing chlorhexidine, those containing polyhexamethylene biguanide, and those containing octenidine. This confirms the recent findings of the comparative antimicrobial efficacy of octenidine and chlorhexidine in saliva (Figure 2). ${ }^{32}$ Thus, octenidine killed streptococci most efficiently, whereas polyhexamethylene biguanide demonstrated superior antifungal activity and control of anaerobic gram-negative $F . n u$ cleatum (Figures 3 and 4 ). The mouth rinse containing $\mathrm{Ci}$ troxx, which consists of natural antimicrobial agents (including bioflavinoids and fruit acids), demonstrated signifcantly weaker antimicrobial efficacy than did the other agents tested. Therefore, its usefulness in antimicrobial mouth rinses is questionable.

Clinical studies have shown that mouth rinses containing octenidine are effective in the control of dental plaque. ${ }^{33,34}$ The efficacy of polyhexamethylene biguanide with regard to bacterial counts, dental plaque, and the 4-day regrowth of plaque has also been investigated. ${ }^{35,36}$ This antimicrobial agent seems to inhibit plaque recolonization and to reduce oral bacterial counts. The present in vitro data are in accordance with previous clinical reports. However, the nature of the present study is not just confirmatory, because the relative efficacy of these novel and commercially available mouth rinses has hitherto not been tested. Although the antimicrobial agents were given, the exact formulas of the mouth rinses were not available. Thus, the mode of action of the Vagents given may have been affected by interactions with other ingredients.

No neutralizing agent was used in the current investigation, which might be a critical issue (because of the possible prolonged effect of the tested agent) that could probably lead to higher observed reduction factors. However, all of the compounds were tested using the exact same assays and test conditions, indicating valid results. In practice, mouth rinses are used for 30 seconds to 1 minute and then are spit out. Most chemical compounds (including chlorhexidine, octenidine, and polyhexamethylene biguanide) are very tenacious, which means that they bind well to tissue (resulting in a depot effect). Within the limitations of our in vitro study, we found the mouth rinses containing octenidine or polyhexamethylene biguanide to be potent alternative oral antiseptics to chlorhexidine.

\section{ACKNOWLEDGMENTS}

We thank Andreas Schötzau from Basel, Switzerland, for his help in performing the statistical analysis.

Potential conflicts of interest. All authors report no conflicts of interest relevant to this article.

Address reprint requests to Andreas F. Widmer, MD, MS, Department of Infectious Diseases and Hospital Epidemiology, University Hospital Basel, Petersgraben 4, Basel CH-4056, Switzerland (awidmer@uhbs.ch).

\section{REFERENCES}

1. Diefenderfer KE, Ahlf RL, Simecek JW, Levine ME. Periodontal health status in a cohort of young US Navy personnel. J Public Health Dent 2007;67:49-54.

2. Dore $P$, Robert $R$, Grollier $G$, et al. Incidence of anaerobes in ventilator- 
associated pneumonia with use of a protected specimen brush. Am J Respir Crit Care Med 1996;153:1292-1298.

3. Verma P. Laboratory diagnosis of anaerobic pleuropulmonary infections. Semin Respir Infect 2000;15:114-118.

4. Chan EY, Ruest A, Meade MO, Cook DJ. Oral decontamination for prevention of pneumonia in mechanically ventilated adults: systematic review and meta-analysis. BMJ 2007;334:889.

5. de Smet AM, Kluytmans JA, Cooper BS, et al. Decontamination of the digestive tract and oropharynx in ICU patients. N Engl J Med 2009;360: 20-31.

6. Davies GE, Francis J, Martin AR, Rose FL, Swain G. 1:6-Di-4'-chlorophenyldiguanidohexane (hibitane); laboratory investigation of a new antibacterial agent of high potency. Br J Pharmacol Chemother 1954;9:192196.

7. Green K, Livingston V, Bowman K, Hull DS. Chlorhexidine effects on corneal epithelium and endothelium. Arch Ophthalmol 1980;98:12731278.

8. Aursnes J. Vestibular damage from chlorhexidine in guinea pigs. Acta Otolaryngol 1981;92:89-100.

9. Henschen $A$, Olson L. Chlorhexidine-induced degeneration of adrenergic nerves. Acta Neuropathol 1984;63:18-23.

10. Stingeni L, Lapomarda V, Lisi P. Occupational hand dermatitis in hospital environments. Contact Dermatitis 1995;33:172-176.

11. Kampf $G$. Effect of chlorhexidine probably overestimated because of lack of neutralization after sampling. Infect Control Hosp Epidemiol 2009;30: 811-812; author reply 812-813.

12. Dettenkofer $M$, Wilson $C$, Gratwohl A, et al. Skin disinfection with octenidine dihydrochloride for central venous catheter site care: a double-blind, randomized, controlled trial. Clin Microbiol Infect 2010;16(6): 600-606.

13. Rosin $M$, Welk A, Bernhardt $O$, et al. Effect of a polyhexamethylene biguanide mouthrinse on bacterial counts and plaque. J Clin Periodontol 2001;28:1121-1126.

14. Pressdispensary. UK company launches health essential for travelling abroad. Citroxx natural biocide helps beat bird flu and other infections. http://www.pressdispensary.co.uk/releases/c99835.php. Accessed October 26, 2009.

15. Mueller SW, Krebsbach LE. Impact of an antimicrobial-impregnated gauze dressing on surgical site infections including methicillin-resistant Staphylococcus aureus infections. Am J Infect Control 2008;36:651-655.

16. Tietz A, Frei R, Dangel M, et al. Octenidine hydrochloride for the care of central venous catheter insertion sites in severely immunocompromised patients. Infect Control Hosp Epidemiol 2005;26:703-707.

17. Pitten FA, Doering $S$, Kramer A, Rosin M. In vitro assay for the screening of the plaque-reducing activity of antimicrobial agents. Arzneimittelforschung 2003;53:182-187.

18. Pitten FA, Werner HP, Kramer A. A standardized test to assess the impact of different organic challenges on the antimicrobial activity of antiseptics. $J$ Hosp Infect 2003;55:108-115.

19. Walter C, Jawor P, Bernimoulin JP, Hägewald S. Moderate effect of enamel matrix derivative (Emdogain Gel) on Porphyromonas gingivalis growth in vitro. Arch Oral Biol 2006;51:171-176.

20. Kazor CE, Mitchell PM, Lee AM, et al. Diversity of bacterial populations on the tongue dorsa of patients with halitosis and healthy patients. $J$ Clin Microbiol 2003;41:558-563.

21. Estes RJ, Meduri GU. The pathogenesis of ventilator-associated pneumonia: I. mechanisms of bacterial transcolonization and airway inoculation. Intensive Care Med 1995;21:365-383.

22. Hamada S, Slade HD. Biology, immunology, and cariogenicity of Streptococcus mutans. Microbiol Rev 1980;44:331-384.

23. Socransky SS, Haffajee AD, Cugini MA, Smith C, Kent RL Jr. Microbial complexes in subgingival plaque. J Clin Periodontol 1998;25:134-144.

24. Teughels W, Kinder Haake S, Sliepen I, et al. Bacteria interfere with $A$. actinomycetemcomitans colonization. J Dent Res 2007;86:611-617.

25. Van Hoogmoed CG, Geertsema-Doornbusch GI, Teughels W, Quirynen M, Busscher HJ, Van der Mei HC. Reduction of periodontal pathogens adhesion by antagonistic strains. Oral Microbiol Immunol 2008;23:43-48.

26. Gmür R, Munson MA, Wade WG. Genotypic and phenotypic characterization of fusobacteria from Chinese and European patients with inflammatory periodontal diseases. Syst Appl Microbiol 2006;29:120-130.

27. Brook I. Fusobacterial infections in children. J Infect 1994;28:155-165.

28. Budtz-Jorgensen E. Hibitane in the treatment of oral candidiasis. J Clin Periodontol 1977;4:117-128.

29. Cannon RD, Chaffin WL. Oral colonization by Candida albicans. Crit Rev Oral Biol Med 1999;10:359-383.

30. Urzua B, Hermosilla G, Gamonal J, et al. Yeast diversity in the oral microbiota of subjects with periodontitis: Candida albicans and Candida dubliniensis colonize the periodontal pockets. Med Mycol 2008;46:783793.

31. Decker EM, Weiger R, von Ohle C, Wiech I, Brecx M. Susceptibility of planktonic versus attached Streptococcus sanguinis cells to chlorhexidine. Clin Oral Investig 2003;7:98-102.

32. Kocak MM, Ozcan S, Kocak S, Topuz O, Erten H. Comparison of the efficacy of three different mouthrinse solutions in decreasing the level of Streptococcus mutans in saliva. Eur J Dent 2009;3:57-61.

33. Beiswanger BB, Mallatt ME, Mau MS, Jackson RD, Hennon DK. The clinical effects of a mouthrinse containing $0.1 \%$ octenidine. J Dent Res 1990;69:454-457.

34. Patters MR, Anerud K, Trummel CL, Kornman KS, Nalbandian J, Robertson PB. Inhibition of plaque formation in humans by octenidine mouthrinse. J Periodontal Res 1983;18:212-219.

35. Rosin M, Welk A, Kocher T, Majic-Todt A, Kramer A, Pitten FA. The effect of a polyhexamethylene biguanide mouthrinse compared to an essential oil rinse and a chlorhexidine rinse on bacterial counts and 4day plaque regrowth. J Clin Periodontol 2002;29:392-399.

36. Welk A, Splieth $\mathrm{CH}$, Schmidt-Martens G, et al. The effect of a polyhexamethylene biguanide mouthrinse compared with a triclosan rinse and a chlorhexidine rinse on bacterial counts and 4-day plaque regrowth. J Clin Periodontol 2005;32:499-505. 